PROCEEDINGS OF THE

AMERICAN MATHEMATICAL SOCIETY

Volume 139, Number 1, January 2011, Pages 1-9

S 0002-9939(2010)10683-4

Article electronically published on September 3, 2010

\title{
THE ŁOJASIEWICZ EXPONENT OF A CONTINUOUS SUBANALYTIC FUNCTION AT AN ISOLATED ZERO
}

\author{
PHẠM TIẾN SƠN
}

(Communicated by Mei-Chi Shaw)

\begin{abstract}
Let $f$ be a continuous subanalytic function defined in a neighborhood of the origin $0 \in \mathbb{R}^{n}$ such that $f$ has an isolated zero at 0 . We describe the smallest possible exponents $\alpha, \beta, \theta$ for which we have the following estimates: $|f(x)| \geq c\|x\|^{\alpha}, \mathfrak{m}_{f}(x) \geq c\|x\|^{\beta}, \mathfrak{m}_{f}(x) \geq c|f(x)|^{\theta}$ for $x$ near zero, where $c>0$ and $\mathfrak{m}_{f}(x)$ is the nonsmooth slope of $f$ at $x$. We prove that $\alpha=\beta+1, \theta=\beta / \alpha$. In the smooth case, we have $\mathfrak{m}_{f}(x)=\|\nabla f(x)\|$, and we therefore retrieve a result of Gwoździewicz, which is a counterpart of the result of Teissier in the complex case.
\end{abstract}

\section{INTRODUCTION}

Let $f:\left(\mathbb{C}^{n}, 0\right) \rightarrow(\mathbb{C}, 0)$ be a complex analytic function with isolated singularity at the origin $0 \in \mathbb{C}^{n}$. It is well known that there exist constants $c, r>0$ and exponents $\beta, \theta$ such that, for all $\|x\| \leq r$,

$$
\|\nabla f(x)\| \geq c\|x\|^{\beta}, \quad\|\nabla f(x)\| \geq c|f(x)|^{\theta} .
$$

Teissier 26] showed that the smallest possible exponents $\beta, \theta$ for (1) are attained along the polar curve of $f$ and satisfy the following relation:

$$
\theta=\frac{\beta}{\beta+1} .
$$

The results of the exact formula of the Łojasiewicz exponent $\beta$ (and hence, $\theta$ ) for weighted homogeneous isolated singularities are in the recent papers by Krasiński, Oleksik and Płoski [16] and by Tan, Yau and Zuo [25] (see also [13, 14]). Estimations of the Łojasiewicz exponent $\beta$ in the general case can be found in [18, [23], [8], 1].

On the other hand, it was shown by Gwoździewicz in [9] that the relation (2) does not necessarily hold for real analytic functions.

Now let $f:\left(\mathbb{R}^{n}, 0\right) \rightarrow(\mathbb{R}, 0)$ be a real analytic function defined in a neighborhood of the origin $0 \in \mathbb{R}^{n}$. Assume that $f(x)>0$ for $0<\|x\| \ll 1$. Then $\nabla f(x)$ is nonzero for $x$ close to the origin. According to the classical Eojasiewicz inequality (see [19,

Received by the editors September 4, 2009.

2010 Mathematics Subject Classification. Primary 14B05; Secondary 32S05.

Key words and phrases. Łojasiewicz exponent, nonsmooth slope, subanalytic function, subdifferential, tangency variety.

This work was supported by NAFOSTED (Vietnam).

(C)2010 American Mathematical Society Reverts to public domain 28 years from publication 
[20, 21]), there exist constants $c, r>0$ and exponents $\alpha, \beta, \theta$ such that, for all $\|x\| \leq r$

$$
|f(x)| \geq c\|x\|^{\alpha}, \quad\|\nabla f(x)\| \geq c\|x\|^{\beta}, \quad\|\nabla f(x)\| \geq c|f(x)|^{\theta} .
$$

Gwoździewicz [9] showed that the best exponents $\alpha, \beta, \theta$ for (3) are attained along the polar curve of $f$, and moreover, these exponents satisfy the following:

$$
\alpha=\beta+1 \quad \text { and } \quad \theta=\frac{\beta}{\beta+1} .
$$

The aim of this paper is to establish a nonsmooth version of the relation (4) for continuous subanalytic functions (Theorem 3.1). See, for example, 2 for the definition and basic properties of subanalytic functions.

Given a continuous subanalytic mapping $f:\left(\mathbb{R}^{n}, 0\right) \rightarrow(\mathbb{R}, 0)$, our approach to generalizing the above-mentioned properties relies on a one-sided notion of generalized gradients called subgradients (see, e.g., 24]). Moreover, our technical tool, contrary to 26] and [9] who use polar curves, is based on the notion of tangency varieties (introduced and studied in [10, [11, [12]).

The paper is organized as follows. In Section 2 we recall some basic notions and facts concerning the Eojasiewicz exponent and the subdifferential. The main result and its proof are given in Section 3 .

\section{Preliminaries}

Throughout this work we shall consider the Euclidean vector space $\mathbb{R}^{n}$ endowed with its canonical scalar product $\langle\cdot, \cdot\rangle$ and we shall denote its associated norm by $\|\cdot\|$.

2.1. The Łojasiewicz exponent. Let us start with the following:

Definition 2.1. By the Eojasiewicz exponent $\ell_{0}(g, h)$ for the inequality $\|g(x)\| \geq$ $c\|h(x)\|^{\alpha}$ we mean the number

$$
\inf \left\{\alpha \in \mathbb{R}_{+} \mid \exists c, r>0\|g(x)\| \geq c\|h(x)\|^{\alpha} \text { for all }\|x\| \leq r\right\} .
$$

We next recall the definition of the order of continuous subanalytic functions (see 9]). Let $g:[0,1) \rightarrow \mathbb{R}$ be a continuous subanalytic function. Here and subsequently we assume that $g \neq 0$ in every neighborhood of zero. Then there exist (see 3 , Lemma 3]) a nonnegative rational number $\nu$ and a continuous function $g_{1}:[0, \delta] \rightarrow$ $\mathbb{R}(0<\delta<1)$ such that for all $\tau \in[0, \delta], g_{1}(\tau) \neq 0$ and $g(\tau)=\tau^{\nu} g_{1}(\tau)$. It is obvious that the exponent $\nu$ is uniquely determined by the function $g$ (even by a germ of $g$ at zero). We call this number the order (at zero) of $g$ and will denote it by $\nu(g)$. We extend the notion of order to subanalytic continuous mappings, putting $\nu(\phi):=\nu(\|\phi\|)$ for $\phi:[0,1) \rightarrow \mathbb{R}^{n}$.

We have the following easy property.

Lemma 2.1. Let $g, h:[0,1) \rightarrow \mathbb{R}$ be continuous subanalytic functions nonvanishing in every neighborhood of zero and let $r$ be a positive rational number. Then

(i) $\nu\left(g^{r}\right)=r \nu(g), \nu(g h)=\nu(g)+\nu(h)$, and

(ii) $\nu(g) \leq \nu(h)$ if and only if there exist $c, \delta>0$ such that $|g(\tau)| \geq c|h(\tau)|$ for all $\tau \in[0, \delta]$.

Proof. See [9, Property 2.1]. 
In the following lemma we reformulate the main result of [3] (see also [2]) in the case of functions with isolated zeros.

Lemma 2.2. Let $U$ be a neighborhood of $0 \in \mathbb{R}^{n}$, and let $g, h: U \rightarrow \mathbb{R}$ be continuous subanalytic functions such that $g^{-1}(0)=h^{-1}(0)=\{0\}$. The following statements hold:

(i) There exists a positive constant c such that $\|g(x)\| \geq c\|h(x)\|^{\ell_{0}(g, h)}$ in a neighborhood of the orgin.

(ii) For every analytic curve $\phi:[0,1) \rightarrow U, \phi(0)=0, \phi \neq 0$, we have $\ell_{0}(g, h) \geq$ $\nu(g \circ \phi) / \nu(h \circ \phi)$.

(iii) There exists an analytic curve $\gamma:[0,1) \rightarrow U, \gamma(0)=0, \gamma \neq 0$, such that $\ell_{0}(g, h)=\nu(g \circ \gamma) / \nu(h \circ \gamma)$.

Proof. See [9, Corollary 2.4].

2.2. The nonsmooth slope. The notion of subdifferential, that is, an appropriate multivalued operator playing the role of the usual gradient mapping, is crucial for our considerations.

Definition 2.2 (see, e.g., 24, Definition 8.3]). (i) The Fréchet subdifferential $\hat{\partial} f(x)$ of a continuous function $f: U \rightarrow \mathbb{R}$ at $x \in U$ is given by

$$
\hat{\partial} f(x):=\left\{v \in \mathbb{R}^{n} \mid \liminf _{y \rightarrow x, y \neq x} \frac{f(y)-f(x)-\langle v, y-x\rangle}{\|y-x\|} \geq 0\right\} .
$$

(ii) The limiting subdifferential at $x \in U$, denoted by $\partial f(x)$, is the set of all cluster points of sequences $\left\{v^{l}\right\}_{l \geq 1}$ such that $v^{l} \in \hat{\partial} f\left(x^{l}\right)$ and $\left(x^{l}, f\left(x^{l}\right)\right) \rightarrow$ $(x, f(x))$ as $l \rightarrow \infty$.

Remark 2.1. It is a well-known result of variational analysis that $\hat{\partial} f(x)$ (and a fortiori $\partial f(x)$ ) is not empty in a dense subset of the domain of $f$ (see 24, for example).

Definition 2.3 (see [5]). Using the limiting subdifferential $\partial f$, we define the nonsmooth slope of $f$ by

$$
\mathfrak{m}_{f}(x):=\inf \{\|v\| \mid v \in \partial f(x)\} .
$$

By definition, $\mathfrak{m}_{f}(x)=+\infty$ whenever $\partial f(x)=\emptyset$.

Remark 2.2. (i) If the function $f$ is of class $C^{1}$, the above notion coincides with the usual concept of the gradient; that is, $\partial f(x)=\hat{\partial} f(x)=\{\nabla f(x)\}$, and hence $\mathfrak{m}_{f}(x)=\|\nabla f(x)\|$.

(ii) Recently, Bolte et al. [5] (see also [17, 4], 6]) proved that if the function $f$ is subanalytic, then the operators $\hat{\partial} f$ and $\partial f$ and the function $\mathfrak{m}_{f}$ are subanalytic. Moreover, the authors have extended the Lojasiewicz gradient inequality to continuous subanalytic functions. Namely (actually their version is more general than the theorem stated below),

Theorem 2.1 ([5, Theorem 3.1]). Let $f:\left(\mathbb{R}^{n}, 0\right) \rightarrow(\mathbb{R}, 0)$ be a continuous subanalytic function defined in a neighborhood of $0 \in \mathbb{R}^{n}$. Then there exist constants $c, r>0$ and an exponent $\theta \in[0,1)$ such that

$$
\left\|\mathfrak{m}_{f}(x)\right\| \geq c|f(x)|^{\theta} \quad \text { for all }\|x\| \leq r .
$$

Note that we have adopted here the following convention: $0^{0}=1$.

The following also is observed by Massey 22. 
Corollary 2.1. Let $G:=\left(g_{1}, \ldots, g_{k}\right):\left(\mathbb{R}^{n}, 0\right) \rightarrow\left(\mathbb{R}^{k}, 0\right)$ be a $C^{1}$-subanalytic mapping defined in a neighborhood of $0 \in \mathbb{R}^{n}$. Then there exist constants $c, r>0$ and an exponent $\theta \in[0,1)$ such that

$$
\sqrt{\left\|\nabla g_{1}(x)\right\|^{2}+\cdots+\left\|\nabla g_{k}(x)\right\|^{2}} \geq c\|G(x)\|^{\theta} \quad \text { for all }\|x\| \leq r .
$$

Proof. Consider the function $f:\left(\mathbb{R}^{n}, 0\right) \rightarrow(\mathbb{R}, 0)$ defined by

$$
f(x):=\max _{i=1, \ldots, k}\left|g_{i}(x)\right| .
$$

Clearly, $f$ is a continuous subanalytic function. Moreover, we have (see [24], for example)

$$
\partial f(x)=\left\{\sum_{i \in I(x)} \lambda_{i} \nabla g_{i}(x) \mid \lambda_{i} \geq 0 \text { and } \sum_{i \in I(x)} \lambda_{i}=1\right\},
$$

where $I(x):=\left\{i \in\{1, \ldots, 2 k\} \mid g_{i}(x)=f(x)\right\}$ and $g_{k+i}(x):=-g_{i}(x), i=1, \ldots, k$. Hence, we have, for all $x \in U$,

$$
\begin{aligned}
\mathfrak{m}_{f}(x) & =\min _{i} \geq 0, \sum_{i \in I(x)} \lambda_{i}=1\left\|\sum_{i \in I(x)} \lambda_{i} \nabla g_{i}(x)\right\| \\
\leq & \left\|\sum_{i \in I(x)} \frac{1}{\# I(x)} \nabla g_{i}(x)\right\|=\frac{1}{\# I(x)}\left\|\sum_{i \in I(x)} \nabla g_{i}(x)\right\| .
\end{aligned}
$$

Applying the Cauchy-Schwarz inequality, we get for some $c>0$ that

$$
\mathfrak{m}_{f}(x) \leq c \sqrt{\sum_{i \in I(x)}\left\|\nabla g_{i}(x)\right\|^{2}} \leq c \sqrt{2 \sum_{i=1}^{k}\left\|\nabla g_{i}(x)\right\|^{2}} .
$$

Then the desired inequality follows at once from Theorem 2.1 .

2.3. The tangency variety. In this paper, we will replace the polar curve by a subanalytic subset of $\mathbb{R}^{n}$ which we call the tangency variety. Precisely, we have the following definition (see also [10, [11, [12]).

Definition 2.4. The tangency variety of a continuous function $f: U \rightarrow \mathbb{R}$ is defined as follows:

$$
\Gamma(f):=\{x \in U \quad \mid \quad \exists \mu \in \mathbb{R} \text { such that } \mu x \in \partial f(x)\} .
$$

Geometrically, in the smooth case, the set $\Gamma(f)$ consists of all points $x \in U$ where the level sets of $f$ are tangent to the sphere in $\mathbb{R}^{n}$ centered at the origin and with radius $\|x\|$.

The following is a simple fact about the tangency variety $\Gamma(f)$.

Property 2.1. Let $f: U \rightarrow \mathbb{R}$ be a continuous function. The origin belongs to the closure of the set $\Gamma(f) \backslash\{0\}$.

Proof. It is sufficient to check that every sphere $\mathbb{S}_{r}^{n-1}:=\left\{x \in \mathbb{R}^{n} \mid\|x\|=r\right\}(0<$ $r \ll 1)$ has a nonempty intersection with $\Gamma(f)$. In fact, since $\mathbb{S}_{r}^{n-1}$ is compact, there exists $x \in \mathbb{S}_{r}^{n-1}$ such that $f(x)=\min _{y \in \mathbb{S}_{r}^{n-1}} f(y)$. It follows from Lagrange's multiplier theorem that $\mu x \in \partial f(x)$ for some $\mu \in \mathbb{R}$; that is, $x \in \Gamma(f)$. 
Remark 2.3. If the continuous function $f$ is subanalytic, then the tangency variety $\Gamma(f)$ is a subanalytic set. The argument is standard (see, e.g., [2]). As we shall not use this statement, we leave the proof as an exercise.

\section{The RESUlt AND ITS PROOF}

The main result of this paper can now be stated as follows.

Theorem 3.1. Let $f: U \rightarrow \mathbb{R}$ be a continuous subanalytic function defined in a neighborhood $U$ of the origin $0 \in \mathbb{R}^{n}$. Assume that $f(x)>0$ for $0<\|x\| \ll 1$. Then there exists an analytic curve $\gamma:[0,1) \rightarrow \Gamma(f), \gamma(0)=0$, for which

(i) the Lojasiewicz exponent $\alpha_{0}$ for the inequality $|f(x)| \geq c\|x\|^{\alpha}$ is equal to $\alpha_{0}=\nu(f \circ \gamma) / \nu(\gamma)$

(ii) the Eojasiewicz exponent $\beta_{0}$ for the inequality $\mathfrak{m}_{f}(x) \geq c\|x\|^{\beta}$ is equal to $\beta_{0}=\nu\left(\mathfrak{m}_{f} \circ \gamma\right) / \nu(\gamma)$

(iii) the Eojasiewicz exponent $\theta_{0}$ for the inequality $\mathfrak{m}_{f}(x) \geq c|f(x)|^{\theta}$ is equal to $\theta_{0}=\nu\left(\mathfrak{m}_{f} \circ \gamma\right) / \nu(f \circ \gamma)$.

Moreover $\alpha_{0}=\beta_{0}+1, \theta_{0}=\beta_{0} / \alpha_{0}$.

Now, we give the following example.

Example 3.1. Let us consider the continuous subanalytic function $f:\left(\mathbb{R}^{2}, 0\right) \rightarrow$ $(\mathbb{R}, 0)$, with $f(x, y):=\max \left\{\left|x^{2}-y^{2}\right|,|x y|\right\}$. Then $f(x, y)>0$ for $(x, y) \neq(0,0)$. Let $\phi:[0,1) \rightarrow \mathbb{R}^{2}$ be an analytic curve defined by $\phi(\tau)=(\tau, \tau)$. We have $f(\phi(\tau))=\tau^{2}$ and $\lim _{\tau \rightarrow 0}\|\phi(\tau)\| /|\tau|=1$. Hence, $\ell_{0}(f,\|\|) \geq 2$. In fact it is easy to check that $\ell_{0}(f,\|\|)=2$. According to Theorem 3.1 we have $\ell_{0}\left(\mathfrak{m}_{f},\|\|\right)=1$ and $\ell_{0}\left(\mathfrak{m}_{f}, f\right)=$ $1 / 2$.

In what follows let $f: U \rightarrow \mathbb{R}$ be a continuous subanalytic function defined in a neighborhood $U$ of $0 \in \mathbb{R}^{n}$.

Remark 3.1. Let $\phi:[0,1) \rightarrow U, \tau \mapsto \phi(\tau)$, be an analytic curve. By definition, $f \circ \phi:[0,1) \rightarrow \mathbb{R}$ is a continuous subanalytic function. Applying the monotonicity lemma (e.g. [27, Theorem 4.1], [7, Theorem 2.1]) to $f \circ \phi$, we deduce that $f \circ \phi$ is absolutely continuous and differentiable (in fact, analytic) in a complement of a finite set.

The proof of Theorem 3.1 will be divided into several steps, which, for convenience, will be called lemmas.

Lemma 3.1. Let $\phi:[0,1) \rightarrow U, \tau \mapsto \phi(\tau)$, be an analytic curve. Then

$$
\left|(f \circ \phi)^{\prime}(\tau)\right| \leq \mathfrak{m}_{f}(\phi(\tau))\left\|\phi^{\prime}(\tau)\right\|,
$$

for all but finitely many $\tau \in[0,1)$.

Proof. Applying the chain rule calculus for the Fréchet subdifferential [24, Theorem 10.6], we get for all but finitely many $\tau \in[0,1)$ that

$$
\begin{aligned}
\hat{\partial}(f \circ \phi)(\tau)=\left\{(f \circ \phi)^{\prime}(\tau)\right\} & =\left\{\frac{d}{d \tau}(f \circ \phi)(\tau)\right\} \\
& =\left\{\left\langle v, \phi^{\prime}(\tau)\right\rangle \mid v \in \hat{\partial} f(\phi(\tau))\right\},
\end{aligned}
$$

which yields via a standard argument that

$$
\left|(f \circ \phi)^{\prime}(\tau)\right| \leq\|v\|\left\|\phi^{\prime}(\tau)\right\| \quad \text { for all } v \in \hat{\partial} f(\phi(\tau)) .
$$


It now follows by passing to the limit (according to Definition 2.2(ii)) that

$$
\left|(f \circ \phi)^{\prime}(\tau)\right| \leq\|v\|\left\|\phi^{\prime}(\tau)\right\| \quad \text { for all } v \in \partial f(\phi(\tau)),
$$

and by taking the infimum over all $v \in \partial f(\phi(\tau))$, we obtain

$$
\left|(f \circ \phi)^{\prime}(\tau)\right| \leq \mathfrak{m}_{f}(\phi(\tau))\left\|\phi^{\prime}(\tau)\right\|
$$

for all $\tau$ in the complement of a finite set.

Lemma 3.2. Under the hypotheses of Theorem 3.1, the function $\mathfrak{m}_{f}(x)$ has an isolated zero at 0 .

Proof. Since $f(x)>0$ for $0<\|x\| \ll 1$, the function $f$ has a strict local minimum at the origin. Thanks to Lagrange's multiplier theorem that $0 \in \partial f(0)$; in particular, $\mathfrak{m}_{f}(0)=0$.

We next claim that the origin is an isolated zero of $\mathfrak{m}_{f}(x)$. By contradiction and using the Curve Selection Lemma (see [15]), there exists an analytic curve $\phi:[0,1) \rightarrow U, \phi(0)=0, \phi \neq 0$, such that $\mathfrak{m}_{f}(\phi(\tau))=0$ for all $\tau \in[0,1)$. By Lemma 3.1, we get for all but finitely many $\tau \in[0,1)$ that

$$
(f \circ \phi)^{\prime}(\tau)=0
$$

It follows that $f(\phi(\tau))=f(\phi(0))=0$ for all $\tau$ in the complement of a finite set, which is a contradiction.

Lemma 3.3. Under the hypothesis of Theorem 3.1, for any analytic curve $\phi:[0,1)$ $\rightarrow U, \phi(0)=0, \phi \neq 0$, we have

(i) $\ell_{0}(f,\|\|) \geq \nu(f \circ \phi) / \nu(\phi)$;

(ii) $\ell_{0}\left(\mathfrak{m}_{f},\|\|\right) \geq \nu\left(\mathfrak{m}_{f} \circ \phi\right) / \nu(\phi)$;

(iii) $\ell_{0}\left(\mathfrak{m}_{f}, f\right) \geq \nu\left(\mathfrak{m}_{f} \circ \phi\right) / \nu(f \circ \phi)$;

(iv) $\nu(f \circ \phi) \geq \nu\left(\mathfrak{m}_{f} \circ \phi\right)+\nu(\phi)$.

Proof. Items (i)-(iii) follow immediately from Lemma 2.2 .

(iv). According to Lemma 3.1 we have

$$
\left|(f \circ \phi)^{\prime}(\tau)\right| \leq \mathfrak{m}_{f}(\phi(\tau))\left\|\phi^{\prime}(\tau)\right\|
$$

for all small $\tau>0$. Combining this with Lemma 2.1, we obtain

$$
\nu\left((f \circ \phi)^{\prime}\right) \geq \nu\left(\mathfrak{m}_{f} \circ \phi\right)+\nu\left(\phi^{\prime}\right),
$$

and hence

$$
\nu(f \circ \phi) \geq \nu\left(\mathfrak{m}_{f} \circ \phi\right)+\nu(\phi) .
$$

Lemma 3.4. Under the hypothesis of Theorem 3.1, there exists an analytic curve $\gamma:[0,1) \rightarrow \Gamma(f), \gamma(0)=0, \gamma \neq 0$, such that the following conditions hold:

(i) $\ell_{0}(f,\|\|)=\nu(f \circ \gamma) / \nu(\gamma)$;

(ii) $\nu(f \circ \gamma)=\nu\left(\mathfrak{m}_{f} \circ \gamma\right)+\nu(\gamma)$.

Proof. Let us consider a ball $K:=\left\{x \in \mathbb{R}^{n} \mid\|x\| \leq r\right\}$ contained in $U$, and put

$$
K^{*}:=\left\{x \in K \mid f(x)=\min _{\|y\|^{2}=\|x\|^{2}} f(y)\right\} .
$$

It is easy to check (see, e.g., [3]) that the set $K^{*}$ is subanalytic and that the origin is a cluster point of $K^{*} \backslash\{0\}$. By the Curve Selection Lemma (see [15]), there exists an analytic curve $\gamma:[0,1) \rightarrow \mathbb{R}^{n}$ such that $\gamma(0)=0$ and $\gamma((0,1)) \subset K^{*} \backslash\{0\}$. Set 
$\alpha:=\nu(f \circ \gamma) / \nu(\|\gamma\|)$. By Lemma 2.1(i), we have $\nu(f \circ \gamma)=\nu\left(\|\gamma\|^{\alpha}\right)$. Thus, by Lemma 2.1(ii), there are positive constants $c, \delta$ such that

$$
f(\gamma(\tau)) \geq c \| \gamma(\tau)) \|^{\alpha} \quad \text { for } \quad \tau \in[0, \delta] .
$$

Since the norm \|\| is a continuous function, there exists $\epsilon>0$ such that $\|x\| \leq$ $\|\gamma(\delta)\|$ for all $\|x\| \leq \epsilon$. Fix $x \in K$ with $\|x\| \leq \epsilon$. By continuity of the function $\tau \mapsto\|\gamma(\tau)\|$, there exists $\tau \in[0, \delta]$ such that $\| \gamma(\tau))\|=\| x \|$. Then it follows from the definition of $K^{*}$ that

$$
f(x) \geq f(\gamma(\tau)) \geq c\|\gamma(\tau)\|^{\alpha}=c\|x\|^{\alpha} .
$$

Therefore

$$
f(x) \geq c\|x\|^{\alpha} \quad \text { for } \quad\|x\| \leq \epsilon .
$$

Consequently, $\ell_{0}(f,\|\|) \leq \alpha$. Then item (i) follows at once from Lemma 3.3(i).

Let us now establish item (ii). Indeed, the properties of $\gamma$ imply that

$$
f(\gamma(\tau))=\min _{\|y\|^{2}=\|\gamma(\tau)\|^{2}} f(y) \quad \text { for } \quad \tau \in[0,1) .
$$

We therefore deduce from Lagrange's multiplier theorem that there exists a function $\mu:[0,1) \rightarrow \mathbb{R}$ such that $\mu(\tau) \gamma(\tau) \in \partial f(\gamma(\tau))$, which means that $\gamma(\tau) \in \Gamma(f)$. By Lemma 3.2. $\mu \neq 0$. Applying the monotonicity lemma (e.g. [27, Theorem 4.1], 77, Theorem 2.1]) to $f \circ \phi$ and the chain rule calculus for the Fréchet subdifferential [24, Theorem 10.6], we get for all but finitely many $\tau \in[0,1)$ that

$$
(f \circ \phi)^{\prime}(\tau)=\left\langle\mu(\tau) \gamma(\tau), \gamma^{\prime}(\tau)\right\rangle=\frac{\mu(\tau)}{2}\left(\|\gamma(\tau)\|^{2}\right)^{\prime} .
$$

Hence

$$
\mathfrak{m}_{f}(\gamma(\tau))=\inf _{v \in \partial f(\gamma(\tau))}\|v\| \leq|\mu(\tau)|\|\gamma(\tau)\|=\frac{2\left|(f \circ \gamma)^{\prime}(\tau)\right|\|\gamma(\tau)\|}{\left(\|\gamma(\tau)\|^{2}\right)^{\prime}}
$$

for all $\tau$ in the complement of a finite set. Combining this with Lemma 2.1, we get

$$
\nu\left(\mathfrak{m}_{f} \circ \gamma\right)+\nu\left(\gamma^{\prime}\right) \geq \nu\left((f \circ \gamma)^{\prime}\right),
$$

which implies that

$$
\nu\left(\mathfrak{m}_{f} \circ \gamma\right)+\nu(\gamma) \geq \nu(f \circ \gamma) .
$$

Then item (ii) follows from Lemma 3.3 (iv).

We are now in a position to finish the proof of Theorem 3.1

Proof of Theorem 3.1. (i). Let $\gamma:[0,1) \rightarrow \Gamma(f)$ be an analytic curve from the statement of Lemma 3.4. Then item (i) follows immediately from Lemma 3.4(i).

(ii). By Lemma 3.2 we may assume (shrinking the ball $U$ if necessary) that $\mathfrak{m}_{f}(x) \neq 0$ for all $x \in U \backslash\{0\}$. Thus, by Lemma 2.2, there exists an analytic curve $\phi:[0,1) \rightarrow U, \phi(0)=0, \phi \neq 0$, for which $\beta_{0}=\frac{\nu\left(\mathfrak{m}_{f} \circ \phi\right)}{\nu(\phi)}$. Using Lemma 3.3 we obtain

$$
\beta_{0}=\frac{\nu\left(\mathfrak{m}_{f} \circ \phi\right)}{\nu(\phi)} \leq \frac{\nu(f \circ \phi)}{\nu(\phi)}-1 \leq \alpha_{0}-1 .
$$

For the curve $\gamma$ we have

$$
\alpha_{0}=\frac{\nu(f \circ \gamma)}{\nu(\gamma)}=\frac{\nu\left(\mathfrak{m}_{f} \circ \gamma\right)}{\nu(\gamma)}+1 \leq \beta_{0}+1
$$

From these inequalities we get $\beta_{0}=\frac{\nu\left(\mathfrak{m}_{f} \circ \gamma\right)}{\nu(\gamma)}=\alpha_{0}-1$. 
(iii). By Lemma 2.2, there exists an analytic curve $\psi:[0,1) \rightarrow U, \psi(0)=0, \psi \neq$ 0 , for which $\theta_{0}=\frac{\nu\left(\mathfrak{m}_{f} \circ \psi\right)}{\nu(f \circ \psi)}$. Using Lemma 3.3 we have

$$
\theta_{0}=\frac{\nu\left(\mathfrak{m}_{f} \circ \psi\right)}{\nu(f \circ \psi)} \leq 1-\frac{\nu(\psi)}{\nu(f \circ \psi)} \leq 1-\frac{1}{\alpha_{0}} .
$$

For the curve $\gamma$ we have

$$
\theta_{0} \geq \frac{\nu\left(\mathfrak{m}_{f} \circ \gamma\right)}{\nu(f \circ \gamma)}=1-\frac{\nu(\gamma)}{\nu(f \circ \gamma)}=1-\frac{1}{\alpha_{0}} .
$$

Collecting together these inequalities we obtain

$$
\theta_{0}=\frac{\nu\left(\mathfrak{m}_{f} \circ \gamma\right)}{\nu(f \circ \gamma)}=1-\frac{1}{\alpha_{0}}=\frac{\beta_{0}}{\alpha_{0}} .
$$

We now suppose that $f$ is a $C^{1}$-subanalytic function. In this case, the definition of the limiting subdifferential implies that $\partial f(x)=\{\nabla f(x)\}$, so that $\mathfrak{m}_{f}(x)=$ $\|\nabla f(x)\|$. Combining these facts with Theorem 3.1, we obtain

Corollary 3.1 ([9, Theorem 1.3]). Let $f: U \rightarrow \mathbb{R}$ be a $C^{1}$-subanalytic function defined in a neighborhood $U$ of the origin $0 \in \mathbb{R}^{n}$. Assume that $f(x)>0$ for $0<\|x\| \ll 1$. Then there exists an analytic curve

$$
\gamma:[0,1) \longrightarrow \Gamma(f)=\left\{x \in \mathbb{R}^{n} \mid \exists \mu \in \mathbb{R} \text { such that } \mu x=\nabla f(x)\right\}, \quad \gamma(0)=0,
$$

for which

(i) the Eojasiewicz exponent $\alpha_{0}$ for the inequality $|f(x)| \geq c\|x\|^{\alpha}$ is equal to $\alpha_{0}=\nu(f \circ \gamma) / \nu(\gamma)$

(ii) the Eojasiewicz exponent $\beta_{0}$ for the inequality $\|\nabla f(x)\| \geq c\|x\|^{\beta}$ is equal to $\beta_{0}=\nu(\operatorname{grad} f \circ \gamma) \nu(\gamma)$

(iii) the Eojasiewicz exponent $\theta_{0}$ for the inequality $\|\nabla f(x)\| \geq c|f(x)|^{\theta}$ is equal to $\theta_{0}=\nu(\operatorname{grad} f \circ \gamma) / \nu(f \circ \gamma)$.

Moreover $\alpha_{0}=\beta_{0}+1, \theta_{0}=\beta_{0} / \alpha_{0}$.

\section{ACKNowledgments}

The author would like to thank the Abdus Salam International Centre for Theoretical Physics, Trieste, Italy, for its hospitality and support.

\section{REFERENCES}

[1] Abderrahmane, O. M.: On the Lojasiewicz exponent and Newton polyhedron, Kodai Math. J., 28 (2005), 106-110. MR2122194 (2006c:32031)

[2] Bierstone, E. and Milman, P. D.: Semianalytic and Subanalytic Sets, Publ. Math. I.H.E.S., 67 (1988), 5-42. MR.972342 (89k:32011)

[3] Bochnak, J. and Risler, J. J.: Sur les exposants de Eojasiewicz, Comment. Math. Helvetici, 50 (1975), 493-507. MR0404674 (53:8474)

[4] Bolte, J., Daniilidis, A. and Lewis, A.: A nonsmooth Morse-Sard theorem for subanalytic functions, J. Math. Anal. Appl., 321 (2006), 729-740. MR.2241151 (2007h:49017)

[5] Bolte, J., Daniilidis A., and Lewis, A.: The Eojasiewicz inequality for nonsmooth subanalytic functions with applications to subgradient dynamical systems, SIAM J. Optim., 17 (2007), 1205-1223. MR 2274510 (2007j:49020)

[6] Bolte, J., Daniilidis, A., Lewis, A. and Shiota, M.: Clarke subgradients of stratifiable functions, SIAM J. Optim., 18 (2007), 556-572. MR2338451 (2008m:49092) 
[7] Coste, M., An introduction to o-minimal geometry, RAAG Notes, Institut de Recherche Mathématiques de Rennes, November, 1999.

[8] Fukui, T.: Eojasiewicz type inequalities and Newton diagrams, Proc. Amer. Math. Soc., 112 (1991), 1169-1183. MR.1065945 (91j:58021)

[9] Gwoździewicz, J.: The Łojasiewicz exponent of an analytic function at an isolated zero, Comment. Math. Helv., 74 (3) (1999), 364-375. MR1710702 (2001d:32037)

[10] Hà, H. V. and Phạm, T. S.: Global optimization of polynomials using the truncated tangency variety and sums of squares, SIAM J. Optim., 19 (2) (2008), 941-951. MR2448921 (2010e:90081)

[11] Hà, H. V. and Pham, T. S.: On the Eojasiewicz exponent at infinity of real polynomials, Ann. Polon. Math., 94 (3) (2008), 197-208. MR2453513 (2009h:14101)

[12] Hà, H. V. and Phạm, T. S.: Solving polynomial optimization problems via the truncated tangency variety and sums of squares, J. Pure Appl. Algebra, 213 (2009), 2167-2176. MR2533315 (2010f:14062)

[13] Haraux, A. and Pham, T. S.: On the Eojasiewicz exponents of quasi-homogeneous functions, preprints of the Laboratoire Jacques-Louis Lions, 2007, Univ. Pierre et Marie Curie, No. R07041 (http://www.ann.jussieu.fr/publications/2007/R07041.pdf).

[14] Haraux, A. and Pham, T. S.: On the gradient of quasi-homogeneous polynomials, preprints of the Laboratoire Jacques-Louis Lions, 2007, Univ. Pierre et Marie Curie, No. R07055 (http://www.ann.jussieu.fr/publications/2007/R07055.pdf).

[15] Hironaka, H.: Subanalytic sets. Number theory, algebraic geometry and commutative algebra, in honor of Y. Akisuki, Kinokuniya, Tokyo, 1973, 435-493. MR0377101 (51:13275)

[16] Krasiński, T., Oleksik, G. and Płoski, A.: The Eojasiewicz exponent of an isolated weighted homogeneous singularity, Proc. Amer. Math. Soc., 137 (2009), 3387-3397. MR2515408 (2010g:32046)

[17] Kurdyka, K.: On gradients of functions definable in o-minimal structures, Ann. Inst. Fourier, 48 (1998), 769-783. MR 1644089 (2000b:03139)

[18] Lichtin, B.: Estimation of Eojasiewicz exponents and Newton polygons, Invent. Math., 64 (1981), 417-429. MR632982 (83b:32006)

[19] Łojasiewicz, S.: Une propriété topologique des sous-ensembles analytiques réels, in Les Équations aux Dérivées Partielles, Éditions du Centre National de la Recherche Scientifique, Paris, 1963, 87-89. MR0160856 (28:4066)

[20] Łojasiewicz, S.: Sur les trajectoires de gradient d'une fonction analytique, Seminari di Geometria 1982-1983 (lecture notes), Dipartemento di Matematica, Univ. Stud. Bologna, 1984, 115-117. MR771152 (86m:58023)

[21] Łojasiewicz, S.: Sur la géométrie semi- et sous-analytique, Ann. Inst. Fourier, 43 (1993), 1575-1595. MR1275210 (96c:32007)

[22] Massey, D. V.: Real analytic Milnor fibrations and a strong Eojasiewicz inequality, preprint, 2009.

[23] Płoski, A.: Sur l'exposant d'une application analytique. I, Bull. Polish Acad. Sci. Math., 32 (1984), 669-673. MR786190 (86j:32025a)

[24] Rockafellar, R. T. and Wets, R.: Variational Analysis, Grundlehren Math. Wiss., 317, Springer, New York, 1998. MR 1491362 (98m:49001)

[25] Tan, S-L., Yau, S. S.-T. and Zuo, H-Q.: Lojasiewicz inequality for weighted homogeneous polynomial with isolated singularity, Proc. Amer. Math. Soc., 138 (2010), 3975-3984.

[26] Teissier, B.: Variétés polaires. I. Invariants polaires des singularités d'hypersurfaces, Invent. Math., 40 (1977), No. 3, 267-292. MR0470246 (57:10004)

[27] van den Dries, L. and Miller, C.: Geometric categories and o-minimal structures, Duke Math. J., 84 (1996), 497-540. MR1404337(97i:32008)

Department of Mathematics, University of Dalat, 1, Phu Dong Thien Vuong, Dalat, VIETNAM

E-mail address: sonpt@dlu.edu.vn 